\section{The eye's mind: brain mapping and psychiatry}

\author{
ED BULLMORE and PAUL FLETCHER
}

Over the past three decades it has become possible to visualise living human brain structure and function in astonishing detail. To pioneering investigators such as Jackson, Meynert, Wernicke, Freud, Alzheimer and Kraepelin our current capacity to image brain structure to the nearest millimetre, and cerebral haemodynamics to the nearest second, would surely have appeared heaven-sent. With these techniques, they might have envisaged a conclusion to their great project of a biological psychopathology, and a resolution of the theoretical questions they first raised about diagnosis and causation of mental disorders.

But this hasn't happened yet. Despite the extraordinary technical developments in neuroimaging (Andreasen, 1997), scepticism is common with respect to its impact on psychiatry. What has imaging told us about schizophrenia, for example, that we did not already know? Why has imaging been largely irrelevant to our understanding of causation in psychiatry? Why has imaging made no difference to the clinician? These are important questions for neuroimaging researchers to address sincerely. After all, the expense of imaging has often been justified by the promise of clinical benefit. We need to be clear about the impact of imaging on psychiatry so far and the prospects for brain mapping and psychiatry in the future. In what follows, we focus predominantly on studies using magnetic resonance imaging (MRI) to measure brain structure or function, because the safety and versatility of MRI make it the pre-eminent form of imaging in psychiatry. However, there is important complementary information to be gained by other methods. Radioligand studies using positron emission tomography (PET) can provide otherwise inaccessible information about receptor density and endogenous transmitter release (Abi-Dargham et al, 2000). Electrophysiological techniques have better temporal resolution than functional MRI (fMRI) and impose a less constrained environment for psychological experiments. One future trend of interest will be the development of integrated or multi-modal imaging techniques, for example combining PET, fMRI and electrophysiological measurements in comprehensive investigations of all accessible aspects of human brain organisation in vivo (Dale $\&$ Halgren, 2001). However, at least in relation to psychiatry, such technical achievements are not necessarily rate-limiting: as we aim to show below, the critical factors have more to do with the 'goodness' of the questions we use imaging to address, which relates to the problems inherent in using 21 st-century methods to investigate the biological correlates of 19th- and early 20th-century nosology.

\section{WHAT HAS IMAGING TOLD US ABOUT SCHIZOPHRENIA, FOR EXAMPLE, THAT WE DID NOT ALREADY KNOW?}

It is now perhaps forgotten that, when the first computed tomography (CT) studies of schizophrenia were reported (Johnstone et al, 1976), psychosis was customarily divided into organic and functional disorders. It was axiomatic that functional psychosis was not associated with organic or structural abnormalities of the brain; yet CT scans showed significant enlargement of the ventricles in people with schizophrenia. This discrepancy was initially negotiated, without jeopardising the nosological orthodoxy of the time, by suggestions that structural brain changes in schizophrenia were incidental or secondary, due to factors such as medication or institutionalisation. However, the intervening years have witnessed the vindication of the contrary view that schizophrenia is indeed characterised by widespread deficits in grey matter and white matter associated with diffuse enlargement of the ventricular system (Wright et al, 2000). Such observations have prompted a renaissance of Wernicke's original idea that psychosis, as well as dysphasia, may be a disorder of associative or integrative functions. To put this in more recent terminology (Mesulam, 2000): we have resumed thinking of psychosis as a disconnection syndrome adversely affecting the structure of largescale neurocognitive networks in the brain (Sigmundsson et al, 2001). This is an important conceptual shift, which (re)locates schizophrenia in the same frame of reference as classical and neoclassical disconnection syndromes in neurology. The shift also makes psychosis accessible and relevant to contemporary systems neuroscience (Pantelis et al, 1997; Goldman-Rakic, 2001). To the extent that imaging has materially contributed to this shift away from the concept of 'functional psychosis', it has already told us something we did not know (or had forgotten) about schizophrenia.

\section{WHAT FACTORS HAVE AFFECTED THE IMPACT OF IMAGING ON PSYCHIATRY?}

Reports of distributed patterns of grey matter deficit in disorders such as attention-deficit hyperactivity disorder (ADHD) (Castellanos et al, 2001; Overmeyer et al, 2001), autism (Courchesne et al, 2001) and various neurogenetic syndromes, prompt the hypothesis that disconnection syndromes might provide a model for the anatomy of neurodevelopmental disorders in general, not solely schizophrenia (Bullmore et al, 1997). However, there is little consensus on which brain regions (or networks) are critically abnormal in any given syndrome. In our view, the most salient reasons for this perceived fuzzyness of the psychiatric imaging literature are issues of sampling, data analysis and study design.

The majority of imaging studies have scanned modest numbers of patients and comparison participants in a cross-sectional case-control design. The study participants are almost never ascertained by random sampling from the relevant populations and may not always be well matched in terms of potentially confounding factors such as age, gender, medication, race, IQ and task performance. Most structural imaging data have been analysed by 'region of interest' morphometry - a labourintensive approach, inevitably unreliable, and tending to focus scientific attention 
perseveratively on the same few brain regions. Functional imaging studies, and more recent structural studies, have broadened the scope of investigation, and made it more reliable, by using computers to warp brain images into the same anatomical space and then to map effects of interest at each and every voxel in the images. Such an approach, however, ushers in the problems of setting an appropriate $P$ value for hypothesis testing when multiple significance tests are conducted simultaneously across many thousands of voxels representing the entire brain.

Altogether, it is easy to see how this set of operating practices could give rise to an internally inconsistent literature, including both false positive results due to unrepresentative samples or uncontrolled confounds and false negative results due to tightly controlled hypothesis testing on small groups. One key to solving this problem is likely to lie in the use of meta-analytical methods to estimate effects of psychiatric disorder on the basis of the pooled results of several imaging studies (Wright et al, 2000). This could be assisted by the creation of digital data repositories, accessible via the internet, available to investigators wanting to apply common methods to amalgamated datasets (Governing Council of the Organization for Human Brain Mapping, 2001). In addition, sensitivity is also likely to be increased through the use of alternative statistical strategies. Computer-intensive data resampling methods can be used in place of asymptotic theory to approximate more exactly the distribution of any test statistic under the null hypothesis (Bullmore et al, 2001; Nichols \& Holmes, 2002). Prior data can be used to condition the analysis of small samples in a Bayesian framework (Friston et al, 2002). Multivariate statistics can be used to describe succinctly distributed systems of mutually correlated regions (Wright et al, 1999; Meyer-Lindenberg et al, 2001). However, larger samples and more-refined analytical methods alone will not address the most fundamental limitation of neuroimaging case-control studies: neither 'caseness' nor 'controllness', defined cross-sectionally, are sufficiently rich descriptions of the manifest cognitive and behavioural variability we wish to relate to the (perhaps even greater) complexity of the brain.

It is widely accepted that the major diagnostic labels subsume a great diversity of symptom profiles, cognitive deficits and drug histories. Each of these aspects of heterogeneity may be associated with variation in brain function and structure. This potentially enormous within-group neurophenotypic variability may mask between-group differences. More radically, it suggests that we might do better to investigate patients defined in terms of symptoms, or dimensions of correlated symptoms, rather than time-honoured diagnostic entities (Liddle, 2001). The most forward-looking examples of this approach have aimed not simply to map a symptomatic disturbance to a physiological or anatomical abnormality but to reformulate the phenomena of psychopathology in terms of normal neurocognitive systems. One example that has proved fruitful is the concept of auditory hallucinations as a manifestation of disordered monitoring of inner speech (Frith, 1996). This model cuts across the diagnostic boundaries between varieties of psychosis. Other comparable recent studies include investigation of negative psychotic symptoms in relation to disordered function of fronto-striato-thalamic circuits (Menon et al, 2001) and characterisation of obsessive and compulsive symptoms in relation to the functional neuroanatomy of disgust and other cardinal emotions (Phillips et al, 2000). If this trend persists, the success of psychiatric imaging research will be accompanied by a subversion of traditional psychiatric diagnoses.

Imaging has already refreshed our awareness that human brain structure and function are sensitive to many factors, especially age and gender, which are nonpathologically variable in the general population. We now need to understand much more exactly how the human brain normally matures and grows old, and whether there are important male/female dimorphisms or racial differences in these normative neurodevelopmental processes. This enhanced understanding of normal neurophenotypic variability over the life cycle will clearly be fundamental to a more precise delineation of developmental and degenerative disorders in terms of abnormal trajectories of brain structural or functional change. Furthermore, the use of longitudinal designs will enrich studies of psychopathological states or incipient disorders, e.g. first episode of psychosis, which may be associated with sub-acute changes in the brain over time (David et al, 1996; Thompson et al, 2001).

\section{WHY HAS IMAGING BEEN LARGELY IRRELEVANT TO OUR UNDERSTANDING OF CAUSATION?}

It might be charged that imaging is nothing but a new phenomenology, substituting one set of theoretically superficial labels, such as 'thought broadcast', with another set of descriptive labels, such as 'hypofrontality'. This is a mistaken view. The progressive trend in imaging is to link symptoms to dysfunctional neurocognitive systems and to link physiological abnormalities in patients to normative principles of large-scale neurophysiological organisation, such as cognitive load-response curves and finite activation capacity (Braver et al, 1997; Fletcher et al, 1998; Callicott et al, 2000). This goes well beyond neophenomenology.

However, it is fair to say that imaging has had little bearing thus far on questions of aetiology and pathogenesis. The most obvious reason for this is, again, experimental design. Simple case-control comparisons will have little to say about aetiology regardless of whether the comparisons are made in terms of psychological test scores, plasma hormone levels or MRI. By the same token there are clearly designs in which imaging could be used more incisively to address causation. One particularly promising strategy is the conjunction of imaging with genetics. This can be done in the context of a classical twin study design, in which nothing is known about the genetic constitution of the participants apart from their zygosity, and the key outcome is some estimate of the heritability of brain structure or function (Wright et al, 2003). This approach can tell us which aspects of the brain are most strongly influenced by genetic effects and, generalised to include a longitudinal dimension, it might also be used to map age-related changes in the genetic control of brain organisation. However, to understand more specifically which genes are critical for which aspects of normal neurophenotypic variability it will be necessary to investigate associations between single gene polymorphisms in the general population and imaging measures of brain structure and function. There are only a few examples of this exciting approach in the literature (Egan et al, 2001; Martinez et al, 2001) - but doubtless there will be more. A complementary approach is the investigation of rare and relatively 
neglected disorders, such as fragile-X syndrome, velo-cardio-facial syndrome, or tuberous sclerosis, which share clearly defined genetic lesions and a risk of psychopathology (Kwon et al, 2001; Ridler et al, 2001; van Amelsvoort et al, 2001). Finally, and most challengingly, we can anticipate multi-centre imaging studies designed on epidemiological principles and appropriately scaled to elucidate the role of genetic or environmental risk factors in broader, multi-factorial syndromes of major public health interest, such as serious mental illness or borderline learning disability.

Crucially, imaging is not inherently uninformative about causation; it can mediate explanatory links between genetic or other risks and cognitive or behavioural outcomes. However, to do this it must - like any other empirical technique - be incorporated in experiments with an ambition to explain causally.

\section{WHY HAS IMAGING MADE NO DIFFERENCE TO CLINICAL PRACTICE?}

There are economic constraints on psychiatric access to neuroimaging. Access to scanners may be rationed to favour examination of patients with medical or surgical conditions considered to be more acute, more life-threatening or remediable. What could imaging possibly tell us about a single psychiatric patient that would be worth the cost of the scan?

We know that extra diagnostic information can be valuable enough in some circumstances, but in general the most useful information we might expect is predictive rather than diagnostic. Is the patient likely to respond to antidepressant or antipsychotic medication with a prolonged latency of clinical effect? Is the patient more likely to benefit from a new, expensive drug than from an older, generic alternative? How likely is the patient to become violent, or to start drinking or taking drugs again? What is the long-term prognosis? These are commonly occurring questions in practice, with definite implications both financially and for clinical management, which we often have to answer vaguely.

We suggest that there is no neuroscientific reason why it should not be possible, in future, for imaging to help us to answer these questions probabilistically instead. For example, fMRI has already been combined with pharmacological challenges to show differences in working memory function related to short-term atypical antipsychotic treatment (Honey et al, 1999 ) and to define abnormalities in frontostriatal response to methylphenidate in children with ADHD (Vaidya et al, 1998). This early evidence suggests that neuroimaging may provide a predictively valuable index of individual characteristics that are inaccessible to purely psychological enquiry. Of course, these individual brain characteristics are likely to be subtle, perhaps only identifiable as quantitative departures from appropriate comparison group norms. Therefore, major advances in the impact of imaging on management of individual patients will probably need to await the creation of large reference databases of brain images acquired from the general population, and widely agreed standards of data analysis, which can be accessed via the internet as a basis for quantitative analysis of the extent to which a patient's image is abnormal or predictive of some clinically important outcome. Admittedly this assumes a level of methodological maturity, infrastructural investment and international cooperation that does not yet exist; but these are details, surely - there remain grounds for optimism.

\section{DECLARATION OF INTEREST}

This work was supported by the Wellcome Trust. E.B. is a shareholder and member of the Scientific Advisory Committee of the Brain Resource Company (http://www.brainresource.com).

\section{REFERENCES}

Abi-Dargham, A., Rodenhiser, J., Printz, D., et al (2000) Increased baseline occupancy of D2 receptors by dopamine in schizophrenia. Proceedings of the National Academy of Sciences of the USA, 97. 8104-8109.

Andreasen, N. C. (1997) Linking mind and brain in the study of mental illness: a project for a scientific psychopathology. Science, 275, 1586-1593.

Braver, T. S., Cohen, J. D., Nystrom, L. E., et al (1997) A parametric study of prefrontal cortex involvement in human working memory. Neurolmage, 5, 49-62.

Bullmore, E. T., Frangou, S. \& Murray, R. M. (1997)

The dysplastic net hypothesis: an integration of developmental and dysconnectionist theories of schizophrenia. Schizophrenia Research, 28, 143-156.

_ , Long, C., Suckling, J., et al (200I) Colored noise and computational inference in neurophysiological (fMRI) time series analysis: resampling methods in time and wavelet domains. Human Brain Mapping, 12, 61-78.
Callicott, J. H., Bertolino, A., Mattay, V. S., et al (2000) Physiological dysfunction of the dorsolateral prefrontal cortex in schizophrenia revisited. Cerebral Cortex, 10, 1078-1092.

Castellanos, F. X., Giedd, J. N., Berquin, P. C., et al (200I) Quantitative brain magnetic resonance imaging in girls with attention deficit/hyperactivity disorder. Archives of General Psychiatry, 58, 289-295.

Courchesne, E., Karns, C. M., Davis, H. R., et al (200I) Unusual brain growth patterns in early life in patients with autistic disorder - an MRI study. Neurology, 57, 245-254.

Dale, A. M. \& Halgren, E. (200I) Spatiotempora mapping of brain activity by integration of multiple imaging modalities. Current Opinion in Neurobiology, II, 202-208.

David, A. S., Woodruff, P. W. R., Howard, R. J., et al (1996) Auditory hallucinations inhibit exogenous activation of auditory association cortex. Neuroreport, 7, 932-936.

Egan, M. F., Goldberg, T. E., Kolachana, B. S., et al (200I) Effect of COMT Val (I08/158) Met genotype on frontal lobe function and risk for schizophrenia. Proceedings of the National Academy of Sciences of the USA, 98, 6917-6922.

Fletcher, P. C., McKenna, P. J., Frith, C. D., et al (1998) Brain activations in schizophrenia during a gradedmemory task studied with functional neuroimaging. Archives of General Psychiatry, 55, 1001-1008.

Friston, K. J., Penny, W., Phillips, C., et al (2002) Classical and Bayesian inference in neuroimaging: overview and theory. Neurolmage, 16, 465-483.

Frith, C. D. (1996) The role of the prefrontal cortex in self-consciousness: the case of auditory hallucinations. Philosophical Transactions of the Royal Society of London Series B, 35I, |505-1512.

Goldman-Rakic, P. (200I) Working memory dysfunction in schizophrenia. In The Frontal Lobes and Neuropsychiatric Illness (eds S. P. Salloway, P. F. Malloy \& J. D. Duffy), pp. 7I-82. Washington, DC: American Psychiatric Publishing.

Governing Council of the Organization for Human Brain Mapping (200I) Neuroimaging databases. Science, 292, 1673-1676; see also http: // www. humanbrainmapping.org.

Honey, G. D., Bullmore, E. T., Soni, W., et al (1999) Differences in frontal cortical activation by a working memory task following substitution of risperidone for typical antipsychotic drugs in schizophrenic patients. Proceedings of the National Academy of Sciences of the USA, 96, 13432-13437.

Johnstone, E. C., Crow, T. J., Frith, C. D., et al (1976) Cerebral ventricular size and cognitive impairment in chronic schizophrenics. Lancet, ii, 924-926.

Kwon, H., Memon, V., Eliez, S., et al (200I) Functional neuroanatomy of visuospatial working memory in fragile $X$ syndrome: relation to behavioral and molecular measures. American Journal of Psychiatry, I58, 1040-105|.

Liddle, P. F. (200I) Disordered Mind and Brain. London: Gaskell.

Martinez, D., Broft, A. \& Laruelle, M. (200I) Imaging neurochemical endophenotypes: promises and pitfalls. Pharmacogenomics, 2, 223-237.

Menon, V., Anagnoson, R. T., Glober, J., et al (200I) Functional magnetic resonance imaging evidence for disrupted basal ganglia function in schizophrenia. American Journal of Psychiatry, 158, 646-649. 
Mesulam, M. M. (2000) Principles of Behavioral and Cognitive Neurology. New York: Oxford University Press.

\section{Meyer-Lindenberg, A., Poline, J. B., Kohn, P. D., et of} (200I) Evidence for abnormal cortical functional connectivity during working memory in schizophrenia. American Journal of Psychiatry, I58, 1809-1817.

Nichols, T. E. \& Holmes, A. P. (2002) Nonparametric permutation tests for functional neuroimaging: a primer with examples. Human Brain Mapping, I5, I-25.

Overmeyer, S., Bullmore, E. T., Suckling, J., et al (200I) Distributed grey and white matter deficits in hyperkinetic disorder: MRI evidence for anatomical abnormality in an attentional network. Psychological Medicine, 3I, 1425-1435.

Pantelis, C., Barnes, T. E., Nelson, H. E., et al (1997) Frontal-striatal cognitive deficits in patients with chronic schizophrenia. Brain, 120, 1823-1843.

Phillips, M. L., Marks, I. M., Senior, C., et al (2000) A differential neural response in obsessive-compulsive disorder patients with washing compared with checking symptoms to disgust. Psychological Medicine, 30, 1037-1050.

Ridler, K., Bullmore, E. T., de Vries, P. J., et al (200I) Widespread anatomical abnormalities of grey and white matter in tuberous sclerosis. Psychological Medicine, 31, 1437-1446.

Sigmundsson, T., Suckling, J., Maier, M., et al (200I) Structural abnormalities in frontal, temporal and limbic

ED BULLMORE, PhD, MRCPsych, PAUL FLETCHER, MRCPsych, Department of Psychiatry, University of Cambridge, UK

Correspondence: Professor Ed Bullmore, Department of Psychiatry, Brain Mapping Unit,

Addenbrooke's Hospital, Hills Road, Cambridge CB2 2QQ, UK. Tel: 0I223 336583/2; fax: 01223 33658I; e-mail: etb23@cam.ac.uk

(First received II March 2002, final revision I5 July 2002, accepted 8 August 2002)

regions and interconnecting white matter tracts in schizophrenia. American Journal of Psychiatry, I58, 234-243.

Thompson, P. M., Vidal, C., Giedd, J. N., et al (200I) Mapping adolescent brain change reveals dynamic wave of accelerated gray matter loss in very early-onset schizophrenia. Proceedings of the National Academy of Sciences of the USA, 98, $11650-11655$.

Vaidya, C. J., Austin, G., Kirkorian, G., et al (1998) Selective effects of methylphenidate in attention deficit hyperactivity disorder: a functional magnetic resonance imaging study. Proceedings of the National Academy of Sciences of the USA, 95, 14494-14499.

van Amelsvoort, T., Daly, E., Robertson, D., et al (200I) Structural brain abnormalities associated with deletion at chromosome 22qII. Quantitative neuroimaging study of adults with velo-cardio-facial syndrome. British Journal of Psychiatry, 178, 412-419.

Wright, I. C., Sharma, T., McGuire, P. K., et al (1999) Supraregional brain systems and the neuropathology of schizophrenia. Cerebral Cortex, 9, 366-378.

_, Rabe-Hesketh, S., Woodruff, P.W. R., et al (2000) Regional brain structure in schizophrenia; a metaanalysis of volumetric MRI studies. American journal of Psychiatry, 157, 16-25.

_, Sham, P., Murray, R. M., et al (2003) Genetic contributions to regional variability in human brain structure: methods and preliminary results. Neurolmage, in press. 